\title{
The Hydrogen Molecule.
}

By Prof. H. Stanley Alren.

$\mathrm{M}^{\mathrm{o}}$ ODELS for the representation and elucidation of physical phenomena have played an important part in the advancement of science. Mathematicians, who employ the method known as "the ignoration of co-ordinates," may be satisfied with either a kinetic or a static model for an atom or a molecule, but the physicist and especially the chemist will, as a rule, prefer a static model. Sir J. J. Thomson ${ }^{1}$ has done much to bridge the gap between chemistry and physics by making a serious attempt to show how, on the electronic theory of matter, atoms may be linked together to form the stable system which constitutes a molecule. To avoid the difficulties inherent in the view that the electrons are in orbital motion, he is led to postulate a more complicated law of force than that of the inverse square of the distance. For example, he considers the result of assuming a repulsion varying inversely as the cube of the distance superposed on the ordinary electrostatic attraction between a positive charge and an electron. Such a law of force may be adjusted to unit, that is, the one hundred millionth part of a centimetre. The distance between the centres of the spheres is 0.53I A.U., with an error of perhaps one or two units in the third significant figure. No physical meaning is to be attached to the size of the spheres themselves.

In a paper published by the Physical Society of London $^{3}$ the writer has pointed out that a force of exactly the type required in Langmuir's theory is provided by the quantum mechanism described by Prof. E. T. Whittaker. ${ }^{4}$ Thus a static atom may be obtained by transferring the motion of the electron in Bohr's atom to the rotation of a "magnetic wheel" in the quantum mechanism. The question then suggested itself whether it might not be possible to obtain a static model of the hydrogen molecule by endowing the nucleus or the electron with a magnetic wheel. After considering various possible cases of this kind which gave models not differing greatly in scale from what might be expected on experimental grounds, it appeared that the simplest and probably

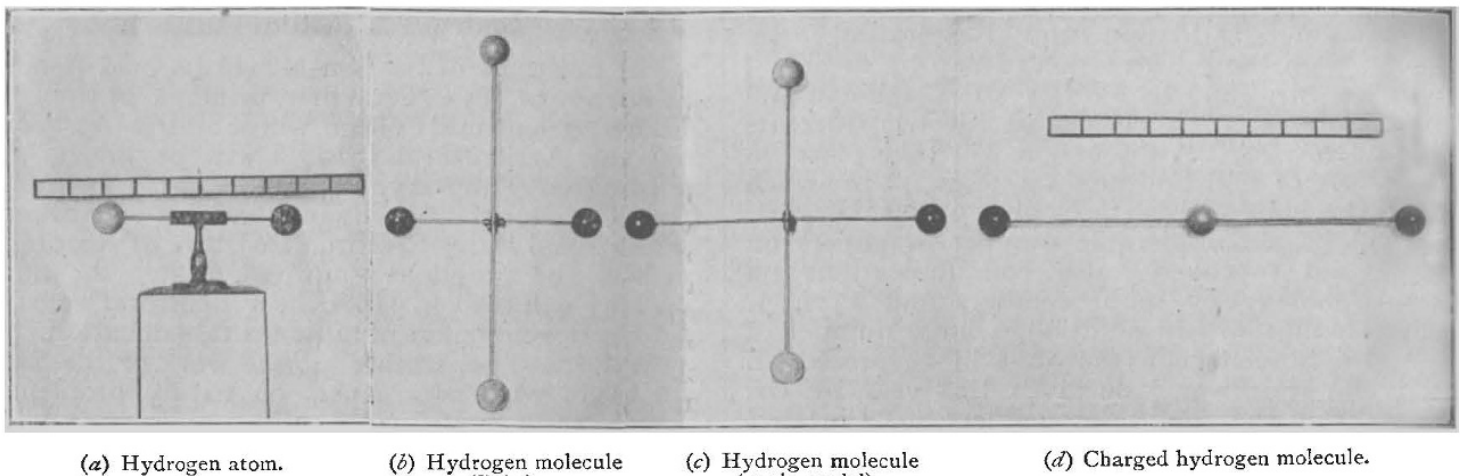

(a) Hydrogen atom.

(b) Hydrogen molecule (Bohr)
Hydrogen molecul (static model)

Fig. I. - Models representing the hydrogen atom and molecule. Black balls represent the positive nuclei and white balls the electrons ; scale above the models = I A. U. (0.0000000I cm.).

meet the requirements of the quantum theory. Dr. Irving Langmuir ${ }^{2}$ has shown that a model of a static hydrogen atom may be obtained possessing many of the properties of the Bohr atom with its circling electrons, if it be assumed that, in addition to the Coulomb force between charged particles, there exists a "quantum force" given by

$$
\mathrm{F}=\frac{\mathrm{I}}{m r^{3}}\left(\frac{n h}{2 \pi}\right)^{2},
$$

acting between an electron (mass $m$, charge $e$ ) and a nucleus. In this formula $n$ is an integer, and $h$ is Planck's constant. When the electrostatic attraction, $e^{2} / r^{2}$, between electron and nucleus is balanced by the repulsion due to the " quantum force," the stationary electron is in stable equilibrium at a distance from the nucleus $r=a_{n}$, where

$$
a_{n}=\frac{\mathrm{I}}{m e^{2}} \cdot\left(\frac{n h}{2 \pi}\right)^{2},
$$

which is the radius of a circular orbit in Bohr's theory of the hydrogen atom. When $n=\mathrm{r}$, we obtain the normal hydrogen atom represented in Fig. I $(a)$, in which the black ball stands for the positive nucleus, or " proton," the white ball for the negative electron. The scale above the model represents one Angström

\footnotetext{
1 Phil. Mag., vol. 4r, p. 5ro, r92r.

2 Phys. Rev., vol. 18, p. 104, 1921.
}

the most accurate results were obtained by postulating the existence of a "quantum force" of the kind introduced by Langmuir, but exerting repulsion or attraction according to the sign of the electrical charges between which the force acts. It will, then, be assumed that in addition to the electrostatic force, $e^{2} / r^{2}$, between elementary charges, there exists a "quantum force"

$$
\mathrm{F}=e^{2} \cdot \frac{a_{n}}{r^{3}},
$$

which is repulsive for unlike charges, but attractive for like charges.

Before considering the configurations obtained on this basis, it will be well to recall the model of the hydrogen molecule devised by Prof. Bohr. This is represented in its most stable form in Fig. I $(b)$ on the same scale as was employed for the hydrogen atom. The two electrons (white balls) spin round in a circular orbit in a plane bisecting at right angles the line joining the hydrogen nuclei. The electrons, which are always at opposite ends of a diameter of the circle, have each an amount of angular momentum, $n h / 2 \pi$, determined by Nicholson's quantum condition. It is easy to show that an electron must be at the vertex of an equilateral triangle having as its base the

3 Proc. Phys. Soc., vol. 34, p. r98, 1922.

4 Proc. Roy. Soc. Edin., vol. 42, p. 129, 1922.

No. 2809 , vor.. I I 2$]$ 
line joining the nuclei, the length of the base being I. I007 $a_{n}$.

It is generally admitted that while the Bohr atom is able to account quantitatively for the phenomena associated with it, the Bohr molecule is far from satisfactory. If a " quantum force" act between each pair of the four electric charges which constitute the neutral molecule, it is possible to replace Bohr's model of the hydrogen molecule by a model with the electrons at rest relatively to the hydrogen nuclei. Various configurations of equilibrium are theoretically possible, but not all of these are stable. The most stable configuration, from the point of view of ordinary statics, appears to be that in which the nuclei and electrons are situated at the corners of a square, with the nuclei at the ends of one diagonal and the electrons at the ends of the other. The length of a diagonal is $\mathrm{I} \cdot 6407 a_{n}$. When $n=x$, the length of a diagonal is $0.87 \mathrm{I}$ Angström units, and this case is illustrated in Fig. I (c). Another configuration agrees exactly with that obtained from Bohr's theory and is shown in Fig. I $(b)$, but the electrons are now at rest instead of in circular motion. In a third configuration, the positions of the charges are similar but the positions of the black and white balls must be interchanged.

It is possible that such configurations might exist for a time side by side, and such a possibility is suggestive in connexion with the varied values sometimes found in determinations of ionisation potentials. When collisions between gaseous molecules are taken into consideration, it is not easy to say what would be the most probable configuration in the final distribution. It is certainly instructive to find such a possibility of different configurations in the case of the simple hydrogen molecule, and points to the necessity of guarding against too rigid an interpretation of the phrase "gaseous molecule" in speaking of more complex gases, whether elements or compounds.

The actual existence of a positively charged hydrogen molecule is demonstrated in experiments by Sir J. J. Thomson and Dr. Aston. On the present hypothesis a stable configuration is obtained by placing the single electron at the middle point of the line joining the atomic nuclei. In the one-quantum state the distance between the nuclei is $\mathrm{I} \cdot 239 \AA$.U., as in Fig. I $(d)$. The ionisation potential for the complete dissociation of the charged molecule is 17.34 volts as against II $\cdot 87$ volts on Bohr's theory. This should serve to discriminate between the two theories.

A possible configuration may be suggested for a neutral triatomic hydrogen molecule, $\mathrm{H}_{3}$, in which the nuclei and electrons are situated at alternate corners of a regular hexagon (length of side, $0.625 \AA$.U.).

Some of the more important numerical data are collected in the following table; full details will appear in a paper in course of publication in the Proceedings of the Royal Society of Edinburgh.

TABLE I.

\begin{tabular}{|c|c|c|c|c|}
\hline & & $d_{1}$ (Å.U.). & $I_{1} \times 10^{4 x}$. & $W$ (volts). \\
\hline \begin{tabular}{l|c} 
Neutral & $\theta$ \\
hydrogen & $45^{\circ}$ \\
$60^{\circ}$ \\
molecule \\
$30^{\circ}$ \\
Ionised molecule \\
Triatomic molecule
\end{tabular} & 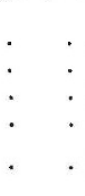 & $\begin{array}{l}0.87 \text { I } \\
0.584_{5} \\
\text { I.0I } \\
\mathrm{I} \cdot 239\end{array}$ & $\begin{array}{r}6.26 \mathrm{I} \\
2.818 \\
8.453 \\
\mathrm{I} 2.66 \\
\left\{\begin{array}{r}9.726 \\
\mathrm{r} 9.452\end{array}\right.\end{array}$ & $\begin{array}{r}30 \cdot 06 \\
29 \cdot 68 \\
29 \cdot 68 \\
17 \cdot 34 \\
\} 46 \cdot 25\end{array}$ \\
\hline
\end{tabular}

$\theta=$ angle between the line joining nucleus and electron and the line joining nuclei.

$d_{1}=$ distance between the nuclei in the one-quantum state.

$\mathrm{I}_{3}=$ moment of inertia in C.G.S. units for the one-quantum state.

$\mathrm{W}=$ work required for complete dissociation expressed in equivalent volts.

The ionisation potentials calculated from the values of $\mathrm{W}$ in the table are in moderately good agreement with the experimental results. A more exacting test of the accuracy of such a model is to be expected from a study of the wave-lengths of lines in the secondary spectrum of hydrogen. Even though the numerical values quoted may have to be modified, it may be claimed that it is now possible to postulate a hydrogen molecule in which the electrons are at rest instead of in orbital motion. It is obvious that the principles here employed may be applied to more complex atomic and molecular systems.

\section{The Liverpool Observatory (Bidston).}

A FTER a career of eighty years, during which the Liverpool Observatory has fulfilled the purpose for which it was designed, the Mersey Docks and Harbour Board, which is responsible for its support and management, has decided that the time has arrived when the usefulness of the institution might be increased by directing its energies into channels additional to those originally contemplated. It may be recalled that the chief objects sought in establishing an Observatory in Liverpool were the communication of accurate time to the Port and the rating of chronometers. The action of the British Association at the Liverpool meeting in I837 contributed largely to the adoption of the necessary measures; the meeting in I923 might give similar encouraging support to the widened programme now under consideration.

The Mersey Docks and Harbour Board considers that the facilities which the Observatory affords for the advancement of knowledge and diffusion of science and learning might be increased if a closer union could be established with the University. As a preliminary measure, it is suggested that the future administration and working of the Observatory may be advantageously entrusted to a joint committee of the Board and University, each nominating five members. This joint committee has now been NO. 2809, VOL. I I 2 ] appointed, the Board's representatives being Mr. C. Livingston, Mr. H. F. Fernie, Col. H. Concanon, the Marine Surveyor and Water Bailiff, the director of the Observatory; and the University nominees, Mr. C. Booth, Prof. Johnstone, Prof. Proudman, and Prof. Wilberforce.

The Dock Board and the University are actuated by a desire to effect an intimate connexion between the recently constituted Tidal Institute, the Observatory, and the department of the Marine Surveyor. The meteorological statistics collected by the Observatory are useful in extending the researches of the Institute in particular directions, while the tidal measurements conducted by the Marine Surveyor afford the necessary means for testing the accuracy of prediction. This closer co-operation has the additional advantage of removing the inconvenience of overlapping.

By utilising the existing establishment as the nucleus of a geophysical observatory, teaching could be combined with research-an extension which is not only feasible but eminently desirable. None of the past activities need be abandoned. The scientific centre would be maintained unimpaired, and its traditions continued. The greater activity exhibited, and the execution of an enlarged programme arranged 